\title{
Influenza Vaccination Programs in Sub-Saharan Africa-Forging a Path for Decision-Making
}

\section{Doudou Diop*}

EPLS Biomedical Research Center, Saint Louis, Senegal

*Corresponding author: Doudou Diop, EPLS Biomedical Research Center, Saint Louis, Senegal, E-mail: deuxdou@gmail.com

Received date: October 03, 2016; Accepted date: October 20, 2016; Published date: October 30, 2016

Copyright: (c) 2016 Diop D. This is an open-access article distributed under the terms of the Creative Commons Attribution License, which permits unrestricted use, distribution, and reproduction in any medium, provided the original author and source are credited.

\begin{abstract}
New Global Burden of Disease estimates attribute nearly $2 \%$ of all-cause mortality during the first five years of life to influenza. Of influenza deaths, $99 \%$ are estimated to occur in low and middle income countries. Effective influenza vaccines have been available for decades, but their use in sub-Saharan Africa has been limited.

Lack of data on seasonal influenza in the region has left questions regarding risk groups and disease burden largely unanswered. Sub-Saharan African countries have limited expertise with data on influenza epidemiology and with influenza vaccines. Such regional capacity must be strengthened if countries are to make the best evidencebased decisions about influenza vaccine use and to maintain a sense of program ownership. A regionalization of efforts would be the most realistic and feasible approach for future influenza vaccine policy consideration as it would leverage individual country strengths and capacities. The pathway for influenza vaccine introduction in sub-Saharan Africa could be as follows: (i) strengthening laboratory capacity and influenza surveillance; (ii) conduct of research studies, such as burden studies, in a few key countries; (iii) improved communication of data; (iv) development of policy strategies; ( $v$ ) building vaccine production capacity; ( vi) identification of financing and development of political will; (vii) and reliance on well-informed technical advisory committees. Additional concerns regarding influenza vaccine implementation include capacity to administer vaccine outside of existing routine immunization systems, program sustainability, and the importance of local and regional program control.
\end{abstract}

Successful introduction of sustained influenza vaccination programs into the sub-Saharan African region will require adherence to a clear plan that includes strong evidence-based decision-making processes, evidence of operational feasibility, and assurances of sustainability.

Keywords: Influenza; Vaccine; Policy; Sub-Saharan Africa

\section{Commentary}

Influenza is one of the major infectious disease threats to the world. New global burden of disease estimates attribute nearly $2 \%$ of all-cause mortality during the first five years of life to influenza [1]. Populations in low and middle income countries like many of those in sub-Saharan Africa are more vulnerable to influenza related complications because of the high prevalence of underlying medical conditions such as HIV infection, tuberculosis (TB), malaria and malnutrition, and limited access to healthcare. Of influenza deaths, $99 \%$ are estimated to occur in low and middle income countries [2]. The optimal way to control the spread of an influenza epidemic and reduce its associated morbidity and mortality is currently through active prophylaxis in the form of vaccination. Effective influenza vaccines have been available for decades, but their use in sub-Saharan Africa has been limited because of questions about the effectiveness, the feasibility of vaccination programs and the cost of the vaccines.

Broadly important factors affecting vaccine adoption are epidemiological considerations, cost-related concerns, the potential programmatic impact on cold chain capacity, and WHO findings on global burden of disease and recommendations.

Large population countries and those with strong academic and research communities are more likely to have access to country- specific data than small population countries. Smaller countries and countries with fewer research institutions and resources to draw on may have to rely on burden of disease information and studies from neighboring countries when country-specific epidemiological data are not available. The prices of new vaccines are, not surprisingly, important to the decision to adopt. However, it is not a simple question of the price per dose or vaccine cost per fully-immunized person, but prices enter into decision making in a multiplicity of interrelated ways, including price levels, variability, fairness, cost comparisons, affordability, and sustainability [3]. For example, South Africa was able to introduce three vaccines over a two-year period with no provision in the budget for the costs of introduction by having vaccine producers make "huge financial contributions" to cover the added costs of social mobilization, expansion of cold chain capacity, and training of health workers on the new vaccines [4]. Currently influenza vaccine is not eligible for the Global Alliance for Vaccines and Immunization (GAVI) subsidy despite the strong recommendation by GAVI for vaccination of pregnant women, for example, that is why the cost of vaccine should be considered in the policy decision-making process for the subSaharan African region. The cost of the vaccine has to be weighed against the benefit. For that reason, it is critical to know the burden of disease and who is most affected. Cost of implementation should be compared to costs of disease in some standardized way to permit comparisons of other intervention/treatment programs for other disease priorities. However, cost of vaccine is only part of the question. The distribution, cold chain, record keeping, keeping vaccine up-to- 
date, and actually administering vaccine is likely to be more expensive. So, cost of vaccine should not stop recommendations being made. Certainly increasing demand should improve competition and decrease costs. Sub-Saharan African countries purchasing as a group may allow for price decreases. Regional manufacturers should be entering the market soon. They will be encouraged to make vaccine and make supply more sustainable financially. An important rationale for giving preference to local production is price, the presumption that local production assures better price and better ability to predict (and to negotiate) future prices, thereby enhancing sustainability [4].

One of the most-often health system component impacted by new vaccine introductions and one of the biggest challenges is the area of vaccine logistics and management. The majority of countries in SubSaharan Africa have insufficient cold storage capacity to adequately handle the issue related to the new vaccine introduction if the vaccine came in one or two-dose vials. However an expansion of national cold chain systems has been noted this last decade in many sub-Saharan African countries with the introduction of pentavalent vaccine and other vaccines like pneumococcal and rotavirus. In some cases the expansion of cold chain capacity took into consideration the introduction of other new vaccines well into the future. However, the cold chain capacity must be always taken in consideration when introducing a new vaccine [4]. The logistics will have to be worked out in small implementation projects.

Perception of vaccine safety is a concern of all countries and it is one of the issues considered by each country's policy makers. WHO prequalification of a vaccine is considered an important guarantee of vaccine safety, and not all countries require further registration of vaccines that have received WHO pre-qualification.

Advocacy can lead to earlier consideration and adoption of new vaccines, if supported by good epidemiological and cost rationale. Training and a specific strategy to deal with negative criticism would be important for the vaccine introduction.

In Sub-Saharan African countries, the Minister of Health plays a critical role. The Minister's role as advocate is very important. However, obtaining agreement from the Ministries of Finance is also critical. Ministries of Finance are important actors to engage with and convince. If you have Minister of Finance support, then new vaccine adoption can be quick.

Sub-Saharan African countries have limited expertise with data on influenza epidemiology and with influenza vaccines. Such regional capacity must be strengthened if countries are to make the best evidence-based decisions about influenza vaccine use and to maintain a sense of program ownership. A regionalization of efforts would be the most realistic and feasible approach for future influenza vaccine policy consideration as it would leverage individual country strengths and capacities.

The pathway for influenza vaccine introduction in Sub-Saharan Africa could be as follows:

\section{Laboratory capacity building}

Countries should set up national influenza-laboratories and/or increase their capacity for laboratory diagnosis of influenza. Sensitive and specific laboratory tests should be used to diagnose influenza virus infection according to international guidelines; such as hemagglutination inhibition (HI), serological assay and RT-PCR. Laboratory capacity at a site to test specimens obviously will facilitate surveillance but is not absolutely necessary if specimens can be tested at a central facility - within the country-as long as the site has the capacity for storage and timely transport.

\section{Influenza surveillance}

The establishment and strengthening of national surveillance capacity supports the long-term goal of generating improved data for national decision-making. The surveillance system should be as streamlined as possible collecting the minimum amount of data needed for decision making. It should be comprised of a small number of surveillance sites that regularly collect and report data from hospitals where the quality of the data can be assured. The sites are chosen to be representative so that the information gathered can be applied to the population as a whole. Ideally, considerations should be given to using explicitly defined influenza-like illness (ILI) and severe acute respiratory illness (SARI) in care settings. To this end, the standardized case definitions or procedures described in the WHO global standard for influenza surveillance [5] issued in May 2012 could be used.

Surveillance of both mild and severe disease contributes to understanding the complete spectrum of influenza illness including differences in the epidemiology of various influenza virus types and subtypes, factors that place individuals at increased risk for severe disease, and the impact that the disease is having on health care delivery systems.

\section{Research: special studies}

Some specific questions regarding influenza transmission are better answered through targeted research projects, which may make use of an existing surveillance platform, rather than attempting to routinely collect large amounts of detail through the entire system. Selected centers of excellence within sub-Saharan Africa could do special studies such as population-based incidence studies, vaccine effectiveness studies to ensure vaccination programs work in African populations or vaccine probe studies to measure the effect of influenza on non-microbiologically confirmed outcomes. Large study of influenza vaccination will need to be conducted by a partnership of academia, government, and industry. Government could provide the public health access, guidance in operations, and policy, as well as some funding for the research. Industry could provide vaccine and guidance in safety evaluation. Academia could provide expertise in innovative design and central coordination of the study. The costeffectiveness is particularly a critical element. The use of effectiveness trials should enable more rational triaging of new vaccines into public health practice by resolving speculative debates about practical costs and benefits [6]. Studies in specific risk groups such as pregnant women as per WHO/SAGE recommendations are also warranted. Other risk groups to be considered, in no specific priority order are: health-care workers, children aged 6-59 months, the elderly and those with high-risk conditions such as severely immunocompromized HIVinfected adults and those with co-morbidities such as tuberculosis. Finally, modeling of the potential impact of influenza vaccine introduction on morbidity and mortality and its economic consequences in the recommended risk groups should be pursued [7].

\section{Generation and communication of data}

The policy-making process will require the synthesis of relevant evidence and effective communication of that evidence to policy- 
Page 3 of 3

makers in specifically developed materials. The research community that generated the evidence-base should connect with the policy community and begin to translate their data into messages and summaries that are consistent with and concordant with health goals. In this step, expertise in communications and policy development is essential.

\section{Development of policy strategies}

The guiding principles of this process are that careful review and consideration of the evidence precede the development of recommendations. The entire process should be transparent, robust and reproducible.

\section{Building vaccine production capacity}

Policy-makers will want to understand the costs involved and likely health impact over time. It is not too early at this point to begin a dialogue with industry to discuss the packaging and presentation requirements that would be needed for a successful introduction in the systems that exist in target countries. For these discussions, forecasting the volume and timing of vaccine demand based on the evidence is critical. Efforts are underway for innovative, lower-cost alternatives to current influenza vaccines by transferring the current technology to developing-country manufacturers or by utilizing new vaccines that can be produced faster and more economically than current technologies. WHO continues to facilitate technology transfer for influenza vaccine production as a major benefit sharing tool.

Identification of financing, development of political will, and reliance on well-informed technical advisory committees

Development of credible, predictable financing policies that overcome the economic obstacles to vaccine use is necessary. The successful vaccine introduction process requires the generation of political will locally to make the implementation of these policies a priority and the capacity of local systems to deliver the vaccine. A broader base of support, beyond immunization systems alone, is often needed to ensure success and build awareness about the larger benefits to overall population health.

\section{Communication and introduction}

An excellent communication strategy is more than needed for this process. The strategy should assist in forging stronger communication links between on-the-ground implementers, development stakeholders and policymakers wherever possible. This will help to improve the flow of timely evidence-based development information, contributing to more effective policy and project design and implementation.

Challenges in disseminating policy information to the public that may include illiteracy, a general lack of understanding of development policy issues and a limited capability or willingness of local media to accurately disseminate such information should be identified very early and addressed. Training and a specific strategy to deal with negative criticism are also critical for this process. Local champion such as pediatricians and other key opinion leaders are central to communication and advocacy. Successful introduction of influenza vaccines into the sub-Saharan African region would also require educational programs and social messaging.

\section{Conclusion}

Influenza historically has been perceived as a mild disease that is uncommon in sub-Saharan Africa. Public health authorities have generally not considered influenza vaccine a high priority, partly because of the cost of the vaccine in relation to other public health vaccines, and the need for annual distribution of influenza vaccine, and also because of having other competing health priorities like malaria, TB, and HIV. Occasionally, local epidemics or global pandemics have drawn policy-makers' attention to immunization needs. Therefore, successful introduction of sustained influenza vaccination programs into the region will require adherence to a clear plan that includes strong evidence-based decision-making processes, evidence of operational feasibility, and assurances of sustainability.

\section{Competing interests}

The authors declare no competing interest.

\section{Disclaimer}

This publication and its contents are solely the responsibility of the author and do not necessarily represent the official views of his organization.

\section{Acknowledgement}

Warm thanks are reserved to PC Faye, JC Victor, J Ortiz, E Montomoli, M Lattanzi, and R Clemens for their guidance.

I gratefully acknowledge the support from the following: I Aboubacar, J Abramson, W Ampofo, AK Banla, F Barondi, AM Bangoura, JA Chapaly, A Clarke, C Cohen, I Dan Dano, A Djassi, J Englund, K Gibba, G Del Giudice, M Griffin, Y Lowe Jallow, H Kadjo, E Langa, M Liwewe, B Maia, P Manangazira, A Martin, M Matonya, MD Mitchell, AW Mounts, P Muthoka, T Ndikumana, K Neuzil, Y Saidu, T Samba, C Sandy, MN Sangare, B Sarkodie, B Schoub, R Seruyange, CT Soumah, S Sow, Z Tarnagda, F Valente, A Wurie, MA Widdowson, A Yam, ERN Yandoko.

\section{References}

1. Lozano R, Naghavi M, Foreman K, Lim S, Shibuya K, et al. (2012) Global and regional mortality from 235 causes of death for 20 age groups in 1990 and 2010: a systematic analysis for the Global Burden of Disease Study. Lancet 380: 2095-2128.

2. Nair H, Brooks WA, Katz M, Roca A, Berkley JA, et al. (2011) Global burden of respiratory infections due to seasonal influenza in young children: a systematic review and meta-analysis. Lancet 378: 1917-1930.

3. World Health Organization. Synthesis report new vaccine adoption in lower-middle income countries. Results for Development Institute (2010).

4. De RD (2011) Views of EPI managers and WHO regional officers on the impact of new vaccine introductions on immunization and health systems. Report of interviews held during the meeting of new and underutilized vaccine initiative (NUVI).

5. World Health Organization. Global standard for influenza surveillance.

6. Clemens J, Brenner R, Rao M, Tafari N, Lowe C, et al. (1996) Evaluating new vacines for developing countries.

7. World Health Organization (2012) Weekly Epidemiology Record 87: 201-216. 